\title{
Habituation of the human blink reflex: The effect of stimulus frequency and the state of arousal
}

\author{
A. J. W. BOELHOUWER \\ Tilburg University, Tilburg, The Netherlands \\ M. GREGORIČ \\ Institute of Clinical Neurophysiology, Ljubljana University, Ljubljana, Yugoslavia \\ and \\ W. E. J. van den BOSCH, L. R. B. SCHOMAKER, and C. H. M. BRUNIA \\ Tilburg University, Tilburg, The Netherlands
}

\begin{abstract}
Electrical stimulation of the supraorbital branch of the human facial nerve resulted in an oligosynaptic ipsilateral reflex $\left(R_{1}\right)$ and a polysynaptic bilateral late reflex $\left(R_{2}\right)$ of the orbicularis oculi muscle. Former studies had already reported different reaction patterns of $R_{1}$ and $R_{2}$ during mental tasks as well as during low-frequency stimulation. The present study was concerned with the possible difference in habituation of $R_{1}$ and $R_{2}$ under conditions with and without a mental task. The habituation paradigm was as follows: Ten control stimuli were presented at 1-min intervals. Identical stimuli were then given during a 30-min session at one of the following frequencies: $1 / 3.75 \mathrm{sec}, 1 / 7.5 \mathrm{sec}, 1 / 15 \mathrm{sec}, 1 / 30 \mathrm{sec}$, and $1 / 60 \mathrm{sec}$, during which time subjects either had a continuous rest or performed a mental task. At the end of this session, the stimulation frequency changed to $1 / 3.75 \mathrm{sec}$. $R_{1}$ increased during a mental task, whereas $R_{2}$ was reduced. It was further concluded that $R_{1}$ underwent more sensitization influences, as $R_{2}$ showed more habituation, and that this divergence was due to differences in central neural pathways.
\end{abstract}

The electrically evoked blink reflex results from stimulation of the supraorbital branch of the fifth cranial nerve. The resulting electromyogram of the orbicularis oculi muscle comprises an early ipsilateral component $\left(R_{1}\right)$ with a latency of $\pm 10 \mathrm{msec}$ and a late bilateral component $\left(R_{2}\right)$ with a latency of \pm 25 40 msec (Kimura, 1973a; Penders \& Delwaide, 1973). Blink reflexes can be modified by presentation of a prestimulus that influences $\mathbf{R}_{\mathbf{1}}$ and $\mathbf{R}_{\mathbf{2}}$ differently (Sanes \& Ison, 1979); that is, an increase of $R_{1}$ and a decrease of $\mathbf{R}_{\mathbf{2}}$ has been observed.

Silverstein, Graham, and Calloway (1980) elicited reflex blinking of the orbicularis oculi muscle using strong white-noise bursts. This resulted in EMG activity comparable to $\mathbf{R}_{\mathbf{2}}$. Also in their experiment, reflex magnitude was reduced by weak prestimulation. Other examples of an opposite behavior of $R_{1}$ and $\mathbf{R}_{\mathbf{2}}$ have been observed with subjects performing

The authors wish to thank Theo Dumoulin and Jan Pijnenburg for their valuable assistance in preparing the final draft. Eugene van Hout ran part of the experiments; Martijn Berger developed the multivariate analyses computer programs. Virginia Differding and Karen Tyson edited and typed the final manuscript. Reprint requests should be addressed to $\mathbf{A}$. J. W. Boelhouwer, Tilburg University, Department of Psychology, P.O. Box 90153, 5000 LE Tilburg, The Netherlands. tasks. Boelhouwer and Brunia $(1977,1979)$ found an increase of $R_{1}$ and a decrease of $R_{2}$ under the influence of a mental task. A different kind of mentaltask influence on $R_{2}$ was reported by Gregorič (1973): a less effective and slower habituation of $R_{2}$ when the subject's attention was distracted from the stimulation by solving arithmetic problems. This resulted in a higher mean value of $\mathbf{R}_{\mathbf{2}}$ during the performance of these mental tasks as compared with a situation involving only rest. The different behavior of $R_{1}$ and $\mathbf{R}_{\mathbf{2}}$ has also been reported for pathological conditions: The observations of Gregoric (1972) and Penders and Delwaide (1971) indicated an absence of $R_{2}$ habituation in parkinsonism; Kimura (1973b) noted the difference in recovery curves of normal subjects and parkinsonian patients. Although the recovery curve of $R_{1}$ is normal in Parkinson's disease, $R_{2}$ showed no suppression, thus suggesting a hyperactivity of the interneurons of $\mathbf{R}_{\mathbf{2}} . \mathbf{R}_{\mathbf{1}}$ was demonstrated as disynaptic in cats (Hiraoka \& Shimamura, 1977) and in humans (Ongerboer de Visser \& Kuypers, 1978), while $\mathbf{R}_{\mathbf{2}}$ had a polysynaptic reflex arc mediated through the trigeminal spinal nucleus with consequent differences in latency between $R_{\mathbf{1}}$ and $\mathbf{R}_{\mathbf{2}}$.

Habituation of exteroceptive reflexes as a result of repeated stimulation is a phenomenon observed in 
many experiments (Desmedt \& Godaux, 1976; Pearson \& Wenkstern, 1972). Thompson and Spencer (1966) reported that habituation depends upon, among other factors, the stimulation frequency. Reflex strength decreases exponentially as a function of the number of stimuli. Application of another (usually stronger) stimulus results in recovery of the habituated response. This sensitization, or dishabituation, is a facilitative process, superimposed on the habituation process. Independently of habituation, given an intrinsic decay time, the dishabituated response gradually decreased to the habituated level (Thompson, Berry, Rinaldi, \& Berger, 1979). These two independent processes conform to two classes of interneurons: some show a decreasing firing frequency after the onset of repeated stimulation; others show an increase followed by a somewhat variable decrement (Groves \& Thompson, 1970).

Habituation of the blink reflex has previously been clearly demonstrated (Boelhouwer \& Brunia, 1979; Gregorič, 1972, 1973; Penders \& Delwaide, 1973; Shahani \& Young, 1968). It can be observed as a diminution of the magnitude of the response, particularly of the bilateral $\mathbf{R}_{\mathbf{2}}$. When stimulation was applied at different frequencies, a magnitude decrement of both the early and the late component was observed: $\mathbf{R}_{\mathbf{2}}$ decreased faster at higher frequencies, whereas the diminution of $R_{1}$ was unrelated to the applied frequency (Boelhouwer \& Brunia, 1979).

The present study was designed to investigate the following: (1) Gregorič (1973) reported that evoking a blink reflex at $1 / 3 \mathrm{sec}$ produced larger $\mathbf{R}_{2}$ components during performance of a mental task as compared with a resting situation, whereas Boelhouwer and Brunia (1977, 1979), stimulating at lower frequencies, observed a decrement of $\mathbf{R}_{\mathbf{2}}$ during performance of a mental task. To study these opposite results, all stimulation frequencies applied by these authors were included in the present study, both during rest and during the performance of a mental task. (2) Does the performance of a mental task delay habituation (Ferrari \& Messina, 1972; Gregorič, 1973)? (3) Will a sudden frequency increase at the end of the experiment result in a different sensitization and/or habituation pattern for both reflex components.

\section{METHOD}

\section{Subjects}

One hundred subjects of both sexes, ranging in age from 18 to 35 years, participated in the experiment. The subjects were paid.

\footnotetext{
Apparatus

During the experimental session, the subject was seated in a dimly lit, electrically shielded soundproof cabin. Blink reflexes were evoked by a Devices isolated stimulator (Type 2533) in series with a Grass CCU-1A constant-current unit. The rectangular pulses of $.10 \mathrm{msec}$ were applied to the supraorbital branch of the 5th cranial nerve above the left eye. Shock intensity was adjusted so that $R_{1}$ was maximal and relatively stable during the time in
}

which approximately 10 test reflexes were evoked with a stimulation frequency of $1 / 10 \mathrm{sec}$. Orbicularis oculi EMG was recorded bipolarly over the inferior orbital portion of both the left and the right muscle from Beckman surface electrodes (infant size), one located $1 \mathrm{~cm}$ below the outer canthus of each eye and the other about $1.5 \mathrm{~cm}$ medially. A ground electrode was located about $1 \mathrm{~cm}$ lateral to the outer canthus of the left eye. Responses were amplified by two Hellige EE preamplifiers (passband .6-1,000 Hz) and recorded on a Hewlett-Packard instrumentation recorder (Type HP 3960, bandpass 0-312 Hz). The EMG was digitized offline (sample frequency $2,048 \mathrm{~Hz}, 12$ bits, input level $\pm 1.5 \mathrm{~V}$ ). The peak-to-peak amplitude of $R_{1}$ and the integral of the ipsilateral and contralateral $\mathbf{R}_{\mathbf{2}}$ (full-wave rectification) were calculated after linear trend removal to correct for possible baseline shifts. Half of the subjects performed a binary choice task. High $(2,400 \mathrm{~Hz})$ and low $(800 \mathrm{~Hz})$ tones were presented with equal probability in random order through a loudspeaker set at a frequency of 60 tones/min. Two response buttons were located on the arms of the chair. The subjects pressed the left button after a low tone and the right one after a high tone. The presentation of the tones was unrelated to the presentation of the electrical stimulus.

\section{Procedure}

The experimental design consisted of three conditions. Condition 1 served to establish a reference baseline level: 10 reflexes were evoked at a frequency of $1 \mathrm{~min}$. Per subject, the mean of these 10 reflexes was considered as the $100 \%$ level. During Condition 2, which lasted $30 \mathrm{~min}$, reflexes were evoked at one of the following five frequencies: $1 / 3.75 \mathrm{sec}, 1 / 7.5 \mathrm{sec}, 1 / 15 \mathrm{sec}$, $1 / 30 \mathrm{sec}$, and $1 / 60 \mathrm{sec}$. In the third condition, 30 reflexes were evoked at a frequency of $1 / 3.75 \mathrm{sec}$. The first and second condition were separated by a break of $5 \mathrm{~min}$. Condition 3 was not separated from Condition 2 . The 100 subjects were randomly divided into five groups of equal size. During Condition 2, one of the above-listed frequencies was used for each group. In addition, throughout Conditions 2 and 3, half of the subjects in each group performed the binary choice task. Participants were also informed about a possible change in stimulation frequency towards the end of Condition 2. This change took place in all but one group, which remained at a stimulation frequency of $1 / 3.75 \mathrm{sec}$ throughout Conditions 2 and 3. This change in frequency was introduced so that the eventual effect of sensitization could be studied.

The percentages of the peak-to-peak amplitudes of $R_{1}$ and the integrals of the ipsilateral and contralateral $\mathbf{R}_{\mathbf{2}}$ were averaged over subjects. The best-fitting negative exponential curves were computed, conforming to the least squares principle, and drawn in the figures for illustrative purposes. Conclusions were based on a multivariate analysis of variance, using the Rao-Khatri model (Timm, 1975; Morrison, 1976). To minimize the error component, percentaged curves of individual subjects were smoothed (smooth length $=3$ ). In order to fulfill statistical assumptions, reduction of the number of dependent measures was necessary. Therefore, the experimental period of $30 \mathrm{~min}$ was subdivided into three periods of $10 \mathrm{~min}$.

\section{RESULTS}

The percentages averaged over subjects in the rest phase, as well as in the mental task phase, resulted in mean curves of the slope of the peak-to-peak amplitudes of $R_{1}$ (Figure 1) and the integrals of $R_{2}$ (Figure 2). For $R_{2}$, only the ipsilateral results are shown. Contralaterally, identical results were found.

\section{Early Reflex}

Mental task. In order to compare the rest and mental task periods (Condition 2) for each stimulation 
frequency, multivariate analyses were applied. During the first $10 \mathrm{~min}, \mathbf{R}_{\mathbf{1}}$ amplitude was larger during the mental task for every frequency except that for the group with $1 / 7.5 \mathrm{sec}$ stimulation frequency (see Table 1); during the second $10 \mathrm{~min}$ of Condition 2, the difference between rest and mental task diminished. Only in the groups experiencing the two lowest frequencies were still larger amplitudes observed during the mental task -an effect completely absent during the third 10 -min period.

Habituation and sensitization. In the beginning of Condition 2, an increase of the peak-to-peak amplitude of $R_{1}$ was observed throughout the rest, as well as during the mental task (Figure 1). The increment during mental tasks was larger; however, the change between Condition 1 (in which the baseline was established) and Condition 2 caused an increase in itself. Sensitization was smallest for the one group which remained at the stimulation frequency of $1 / 60 \mathrm{sec}$ throughout Conditions 1 and 2. All other groups manifested a higher degree of sensitization: that is, the number of reflex amplitudes above the baseline in Condition 2 was larger for these groups. The greatest degree of sensitization could be seen in the group with the highest frequency changeover between Condition 1 and Condition $2(1 / 60 \mathrm{sec}$ to $1 / 3.75 \mathrm{sec}$ ).

The frequency change that took place between Conditions 2 and 3 of the experiment also caused sensitization, and this was most pronounced in the group that moved from a stimulation frequency of $1 / 60 \mathrm{sec}$ to one of $1 / 3.75 \mathrm{sec}$, that is, the group experiencing the largest changeover. (Note that one group remained at a stimulation frequency of $1 / 3.75 \mathrm{sec}$ throughout Conditions 2 and 3.)

The decrement taking place during the 30 min of

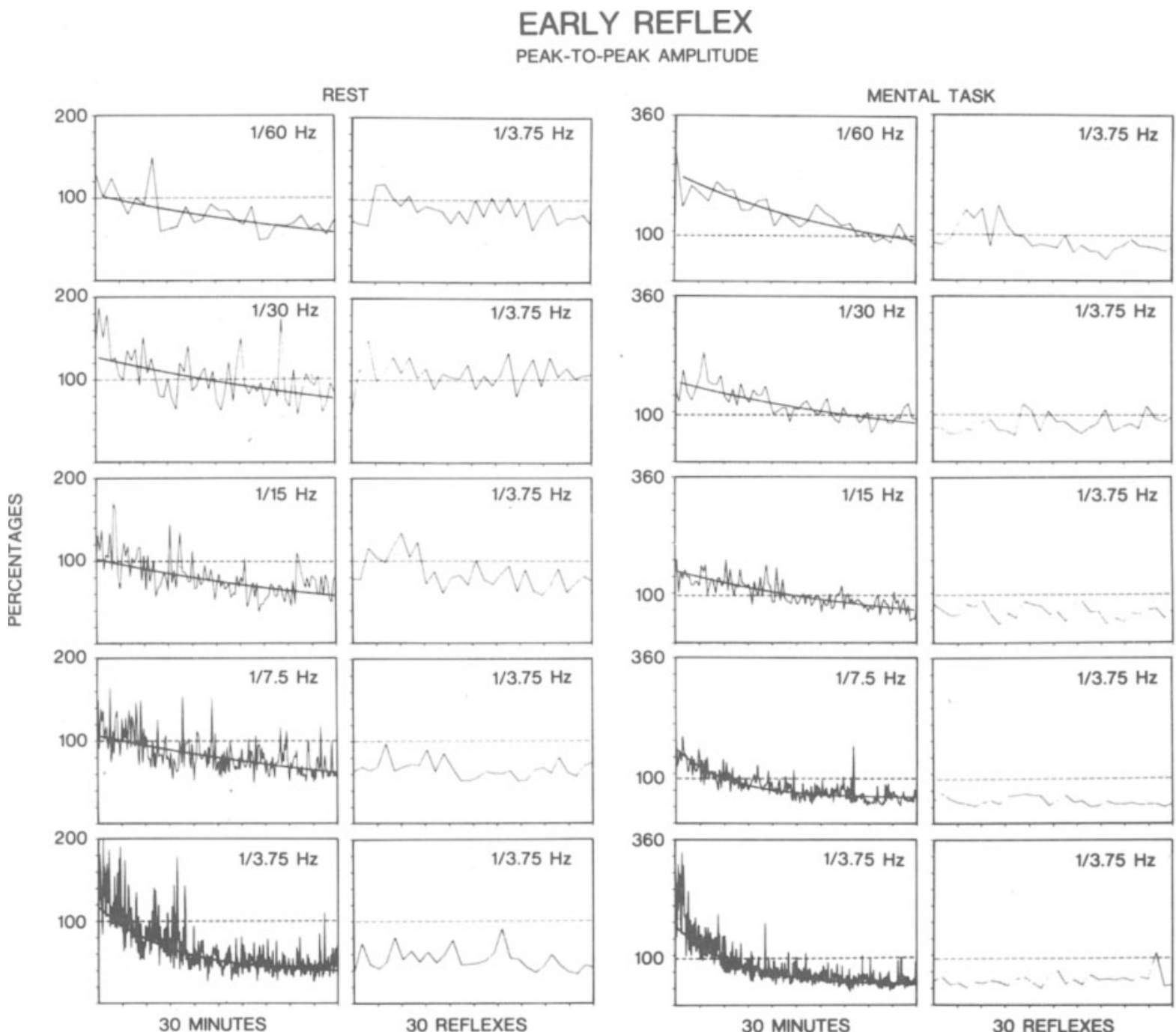

Figure 1. The course of peak-to-peak amplitudes of $R_{1}$ during a rest (left) and during performance of a mental task (right) at different stimulation frequencies. After $30 \mathrm{~min}, 30$ reflexes were evoked at the highest stimulation frequency (1/3.75 sec). Reflex amplitudes are expressed as a percentage of the mean of 10 control reflexes evoked at a frequency of $1 / 60$ sec. 
Condition 2 seems to be independent of the applied frequency. That is, for the participants performing a task, as well as those who rested, a higher frequency only slightly influenced the faster decrement during this period (see Figure 1). This was confirmed through application of a multivariate analysis that showed that within the rest and mental task condition, the frequency slopes were parallel (Wilks $\lambda=.372$ during the rest; $\lambda=.443$ during the task).

\section{Late Reflex}

Mental task. Multivariate analyses were applied to compare the rest and mental task during Condition 2 for each stimulation frequency (Table 1). In general, the $\mathbf{R}_{\mathbf{2}}$ integral for those participants performing a task was smaller than or equal to that of the participants who rested; however, two exceptions were noted. During the first 10 min of Condition 2, participants performing a task in the groups experiencing a stimulation frequency of $1 / 60 \mathrm{sec}$ or $1 / 15 \mathrm{sec}$ evidenced a smaller $\mathbf{R}_{\mathbf{2}}$. Furthermore, the $\mathbf{R}_{\mathbf{2}}$ integral for those performing a task at a stimulation frequency of $1 / 3.75 \mathrm{sec}$ in all three phases of Condition 2 was larger (see Table 1 and Figure 2).

Habituation and sensitization. In contrast with $R_{1}$, no increase in $R_{2}$ was observed at the beginning of Condition 2. The decrement of the late component during rest was dependent on the applied frequency (Figure 2). The significant effect of nonparallel slopes during the first $10 \mathrm{~min}$ of the rest (Condition 2) confirmed this (Wilks $\lambda=.265, \mathrm{p}<.01$ ). The larger the frequency changeover at the end of the experiment, the more pronounced was habituation at the $1 / 3.75$-sec frequency of Condition 3. Although direct comparison of habituation after Condition 1 and after Condition 2 is difficult (given the difference in time scale), it should be noted that there is an equal pattern of $\mathbf{R}_{\mathbf{2}}$ in the group with stimulation frequency changing from $1 / 60 \mathrm{sec}$ to $1 / 3.75 \mathrm{sec}$ at the start of Condition 2 and in the group with stimulation frequency changing from $1 / 60 \mathrm{sec}$ to $1 / 3.75 \mathrm{sec}$ after Condition 2 (see Figure 2).

\section{DISCUSSION}

By comparing the effect of habituation and sensitization, as well as the influence of a mental task, it can be seen from this study that the early and late component of the blink reflex behave differently.

\section{Mental Task}

The increase in the amplitude of $R_{1}$ during the performance of a mental task is presumably due to the higher arousal level. This phenomenon has also been observed in other reflex studies. An increase of Achilles tendon reflex amplitudes (Brunia, 1971) during a binary choice task and of $R_{1}$ of the blink reflex during the same task (Boelhouwer \& Brunia, 1977, 1979) has been observed. The polysynaptic reflex of the biceps femoris muscle in response to stimulation of the group II skin afferents was also larger during different kinds of mental tasks, as was the H-reflex, in some studies (Bathien \& Hugelin, 1969).

As far as the late component of the blink reflex is concerned, results opposite to $R_{1}$ have often been reported. One of the purposes of this study was to confirm the different results of Boelhouwer and Brunia $(1977,1979)$, who reported a decrement of $R_{2}$ during a mental task, and of Gregorič (1973), who found an increase of $\mathbf{R}_{\mathbf{2}}$ during a mental arithmetic task. The results of the present study indeed cover both opposite opinions. At the highest stimulation frequency $(1 / 3.75 \mathrm{sec})$, comparable to that of Gregoric (1973), a sharp decrease was seen at rest, while during the mental task habituation was delayed. Comparing rest and task at the 1/3.75-sec stimulation frequency resulted in the conclusion that $\mathbf{R}_{\mathbf{2}}$ was larger during a mental task. However, at the other stimulation fre-

Table 1

Multivariate Hypothesis Testing for Coincidence (C) and Parallelism (P) During Three Periods of 10 Min for the Rest vs. Task Conditions of R1 and R2, Respectively

\begin{tabular}{|c|c|c|c|c|c|c|}
\hline \multirow{2}{*}{$\begin{array}{c}\text { Stimulation } \\
\text { Frequency }\end{array}$} & \multicolumn{2}{|c|}{ First $10 \mathrm{Min}$} & \multicolumn{2}{|c|}{ Second $10 \mathrm{Min}$} & \multicolumn{2}{|c|}{ Third $10 \mathrm{Min}$} \\
\hline & $\mathrm{C}$ & $\mathbf{P}$ & $\mathrm{C}$ & $\mathbf{P}$ & $\mathrm{C}$ & $\mathbf{P}$ \\
\hline \multicolumn{7}{|c|}{ Early Reflex } \\
\hline $\begin{array}{l}1 / 60 \\
1 / 30 \\
1 / 15 \\
1 / 7.5 \\
1 / 3.75\end{array}$ & $\begin{array}{l}.230 * \dagger \\
.246 * \dagger \\
.186 * \dagger \\
.364 \\
.238 * \dagger\end{array}$ & $\begin{array}{l}.458 \\
.266^{*} \\
.423 \\
.470 \\
.242^{*}\end{array}$ & $\begin{array}{l}.200 * \dagger \\
.229 * \dagger \\
.312 \\
.426 \\
.773\end{array}$ & $\begin{array}{l}.456 \\
.252 * \\
.315 \\
.445 \\
.783\end{array}$ & $\begin{array}{l}.547 \\
.591 \\
.631 \\
.504 \\
.997\end{array}$ & $\begin{array}{l}.546 \\
.711 \\
.673 \\
.579 \\
.836\end{array}$ \\
\hline \multicolumn{7}{|c|}{ Late Reflex } \\
\hline $\begin{array}{l}1 / 60 \\
1 / 30 \\
1 / 15 \\
1 / 7.5 \\
1 / 3.75\end{array}$ & $\begin{array}{l}.220 * \dagger \dagger \\
.704 \\
.189 * \dagger \dagger \\
.580 \\
.142 * * \dagger\end{array}$ & $\begin{array}{l}.542 \\
.737 \\
.211 * \\
.632 \\
.162 * *\end{array}$ & $\begin{array}{l}.605 \\
.656 \\
.558 \\
.369 \\
.126 * * \dagger\end{array}$ & $\begin{array}{l}.677 \\
.668 \\
.721 \\
.471 \\
.171 * *\end{array}$ & $\begin{array}{l}.111^{* * \dagger \dagger} \\
.754 \\
.439 \\
.566 \\
.075^{* * \dagger}\end{array}$ & $\begin{array}{l}.167^{* *} \\
.776 \\
.526 \\
.616 \\
.146^{* *}\end{array}$ \\
\hline
\end{tabular}

Note-Stimulation frequency is given in Hertz. A significant Wilks $\lambda$ means rejection of the aforementioned hypotheses.

${ }^{*} p<.05 . \quad{ }^{*} p<.01$. tTask $>$ rest. HTask $<$ rest. 


\section{LATE REFLEX}

INTEGRAL.

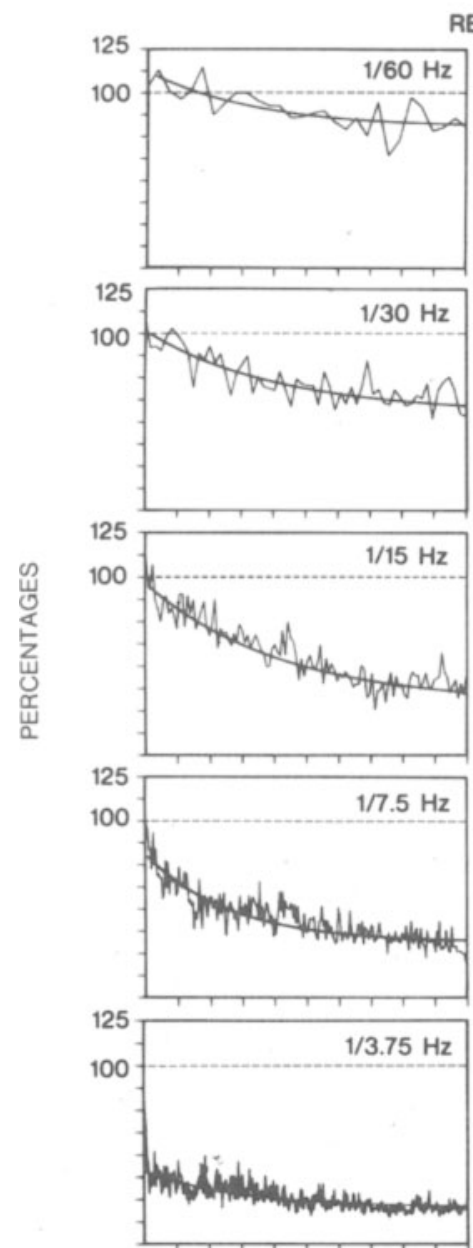

30 MINUTES
REST
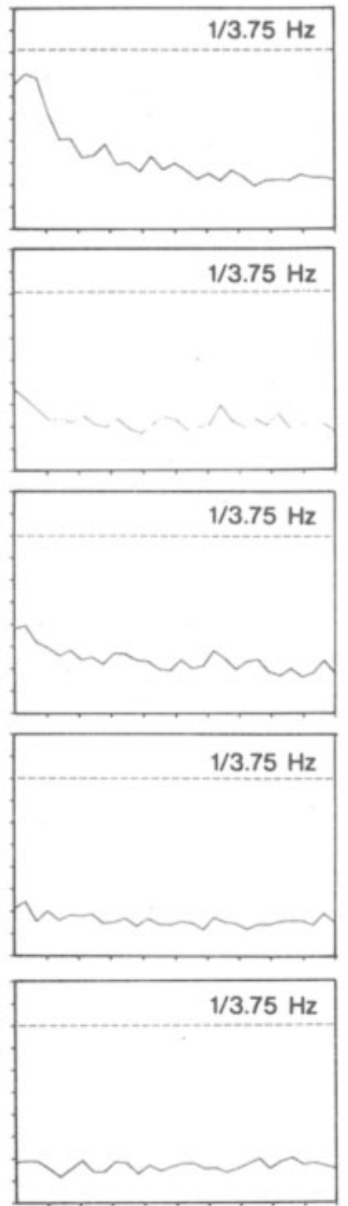

30 REFLEXES
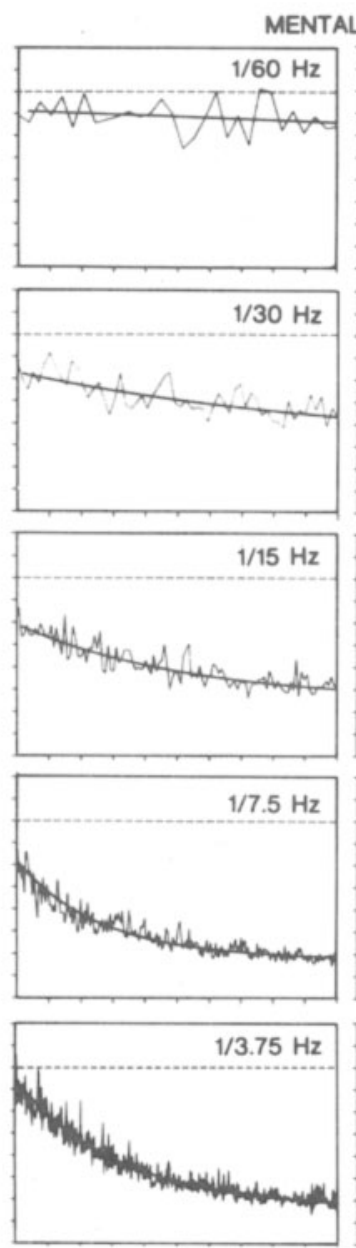

30 MINUTES
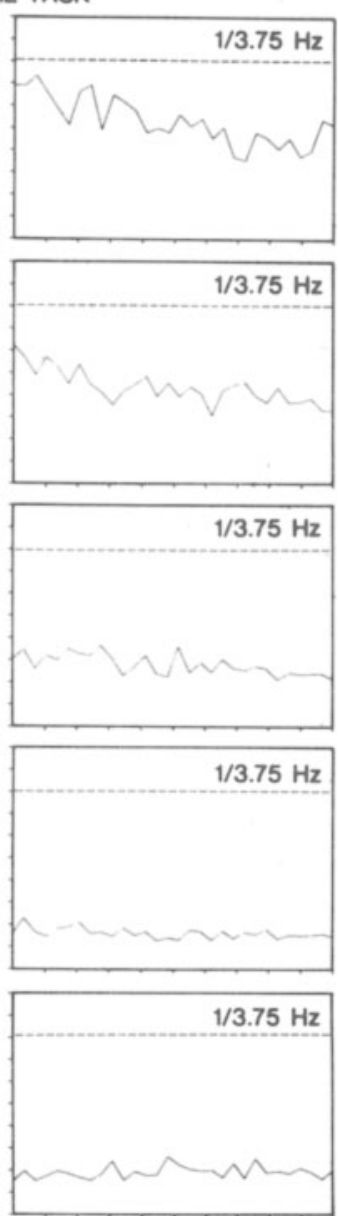

30 REFLEXES

Figure 2. The course of the integrals of $R_{2}$ during a rest (left) and during performance of a mental task (right) at different stimulation frequencies. After $30 \mathrm{~min}, 30$ reflexes were evoked at the highest stimulation frequency $(1 / 3.75 \mathrm{sec})$. Integrals are expressed as a percentage of the mean of 10 control reflexes evoked at a frequency of $1 / 60$ sec.

quencies, the delayed habituation during task performance was not seen, leading to the conclusion that $\mathbf{R}_{\mathbf{2}}$ was reduced during mental task. In Conditions 2 and $3, R_{2}$ showed values below the $100 \%$ baseline level, so the actual conclusion at the highest stimulation frequency $(1 / 3.75 \mathrm{sec})$ was that $\mathbf{R}_{2}$ was not larger during task, because it remained closest to the $100 \%$ baseline. The conclusion reached by Ferrari and Messina (1972) and by Gregorič (1973)-that a mental task delayed habituation of $R_{2}$-held for the highest stimulation frequency.

\section{Habituation and Sensitization}

$\mathbf{R}_{\mathbf{1}}$ and $\mathbf{R}_{\mathbf{2}}$ also differed as far as habituation and sensitization are concerned. The amplitude of the early component in Condition 2 was always above the $100 \%$ baseline level. The changeover between the baseline condition (1/60 sec) and Condition 2 caused the clearest sensitization at the highest frequency change. This phenomenon could also be seen at the end of the experiment, after the frequency change of Condition $3(1 / 3.75 \mathrm{sec})$. The classical theoretical curve of Groves and Thompson (1970) showed habituation and sensitization to be different physiological processes. $R_{1}$ seemed to confirm this curve as far as the initial increment followed by a decrease was concerned. In Condition 2, the slopes of $\mathbf{R}_{\mathbf{1}}$ amplitudes for all frequencies during rest were parallel, and this same phenomenon held true for all frequencies during task performance. The decrease of $R_{1}$ was therefore concluded to be independent of the applied frequency. Boelhouwer and Brunia (1979) also found the decrease of $R_{1}$ in their experiments to be relatively independent of the applied frequencies. This finding 
runs contrary to the frequency-dependent criterium of habituation as formulated by Groves and Thompson (1970) and Thompson and Spencer (1966). Although some habituation of $R_{1}$ was seen in the present experiment (i.e., the decrement below the $100 \%$ baseline level), the present results could indicate that the $R_{1}$ reflex circuitry was influenced more by sensitizationtype neurons than by habituation-type neurons.

$\mathbf{R}_{2}$, on the contrary, showed no sensitization; that is, no initial increment of $\mathbf{R}_{\mathbf{2}}$ was seen in the curves. The habituation of $\mathbf{R}_{\mathbf{2}}$ was dependent on the stimulation frequency. The change from Condition 2 to Condition 3, even in the frequency change from $1 / 60 \mathrm{sec}$ to $1 / 3.75 \mathrm{sec}$, caused no visible sensitization. Therefore, the conclusion seems warranted that the reflex loop of $\mathbf{R}_{2}$ underwent more habituation influences.

The differences between $R_{1}$ and $R_{2}$ are of central origin, as both components have a common afferent and efferent pathway, that is, the trigeminal and facial nerves. The central circuit of $R_{1}$ is located in the pons. It probably contains one interneuron, located between the principle sensory nucleus of the fifth nerve and the motor nucleus of the seventh nerve (Hiraoka \& Shimamura, 1977). Recent studies of Holstege and Graveland (1980) with cats pointed to the involvement of another cell group in the $R_{1}$ circuitry. This " $R_{1}$ nucleus" was thought to be located in the ventrolateral reticular formation just caudal to the trigeminal motor nucleus. The $\mathbf{R}_{\mathbf{2}}$ circuitry is polysynaptic, descending into the lower medulla and crossing at the level of the obex. The exact localization of the $\mathbf{R}_{\mathbf{2}}$ circuitry is even more speculative than that of $R_{1}$. In the present experiment, $R_{1}$ was found to reflect sensitization, as well as a change in the arousal level. It is not clear whether these two phenomena are based on a process taking place in the reflex circuitry of $R_{1}$ itself, or on the many cortical and subcortical influences that are known to act on the brainstem level. The same question holds true for $\mathbf{R}_{2}$, although influences of higher structures are even more plausible in a polysynaptic reflex loop.

\section{REFERENCES}

Bathien, N., \& Hugelin, A. Réflexes monosynaptiques et polysynaptiques de l'homme au cours de l'attention. Electroencephalography and Clinical Neurophysiology, 1969, 26, 604-612.

Boelhouwer, A. J. W., \& Brunia, C. H. M. Blink reflexes and the state of arousal. Journal of Neurology, Neurosurgery and Psychiatry, 1977, 40, 58-63.

Boelhouwer, A. J. W., \& Brunia, C. H. M. Effect of stimulus frequency on the blink reflex during rest and a task. Electroencephalography and Clinical Neurophysiology, 1979, 47, 711-716.

Brunia, C. H. M. The influence of a task on the Achilles tendon and Hoffman reflex. Physiology \& Behavior, 1971, 6, 367-373.

Desmedt, J. E., \& Godaux, E. Habituation of exteroceptive suppression and of exteroceptive reflexes in man as influenced by voluntary contraction. Brain Research, 1976, 106, 21-29.

Ferrari, E., \& Messina, C. Blink reflexes during sleep and wakefulness in man. Electroencephalography and Clinical Neurophysiology, 1972, 32, 55-62.

Gregorič, M. Neurophysiological analysis of the blink reflex in parkinsonism. Akademija Nauka I Umjetnosti Bosni I Hercegovine. Posebna Indanja XVII (Academy of Sciences and Arts of Bosni and Hercegovine, Special Issue XVII), 1972, Serajewo, 219-227.

GreGorič, M. Habituation of the blink reflex: Role of selective attention. In J. E. Desmedt (Ed.), New developments in electromyography and clinical neurophysiology (Vol. 3). Basel: Karger, 1973.

Groves, P. M., \& Thompson, R. F. Habituation: A dual-process theory. Psychological Review, 1970, 77, 419-450.

Hiraoka, M., \& Shimamura, M. Neural mechanisms of the corneal blinking reflex in cats. Brain Research, 1977, 125, 265-275.

Holstege, G., \& Graveland, G. A. An autoradiographical and HRP study about the anatomical substrate of the blink reflex in the cat. Neuroscience Letters, 1980, Suppl. 5, 337.

KimurA, J. The blink reflex as a test for brain-stem and higher central nervous system function. In J. E. Desmedt (Ed.), New developments in electromyography and clinical neurophysiology (Vol. 3). Basel: Karger, 1973. (a)

KimuRA, J. Disorder of interneurons in parkinsonism: The orbicularis oculi reflex to paired stimuli. Brain, 1973, 96, 87-96. (b)

Morrison, D. F. Multivariate statistical methods. New York: McGraw-Hill, 1976.

Ongerboer de Visser, B. W., \& KuYpers, H. G. J. M. Late blink reflex changes in lateral medullary lesions. An electrophysiological and neuro-anatomical study of Wallenberg's syndrome. Brain, 1978, 101, 285-294.

Pearson, J. A., \& Wenkstern, B. Habituation and sensitization of the flexor withdrawal reflex. Brain Research, 1972, 43, 107-118.

Penders, J. A., \& Delwaide, P. J. Blink reflex studies in patients with parkinsonism before and during therapy. Journal of Neurology, Neurosurgery and Psychiatry, 1971, 34, 674-678.

Penders, J. A., \& Delwaide, P. J. Physiologic approach to the human blink reflex. In J. E. Desmedt (Ed.), New developments in electromyography and clinical neurophysiology (Vol. 3). Basel: Kruger, 1973.

SANES, J. N., \& Ison, J. R. Conditioning auditory stimuli and the cutaneous eye blink reflex in humans: Differential effects according to oligosynaptic or polysynaptic central pathways. Electroencephalography and Clinical Neurophysiology, 1979, 47, 546-555.

Shahani, B., \& Young, R. R. A note on blink reflexes. Journal of Physiology (London), 1968, 198, 103-104.

Silverstein, L. D., Graham, F. K., \& Calloway, J. M. Preconditioning and excitability of the human orbicularis oculi reflex as a function of state. Electroencephalography and Clinical Neurophysiology, 1980, 48, 406-417.

Thompson, R. F., Berry, S. D., Rinaldi, P. C., \& Berger, T. W. Habituation and the orienting reflex: The dual-process theory revisited. In H. D. Kimmel, E. H. van Olst, J. H. Orlebeke (Eds.), The orienting reflex in humans. Hillsdale: Erlbaum, 1979.

Thompson, R. F., \& Spencer, W. A. Habituation: A model phenomenon for the study of neuronal substrates of behavior. Psychological Review, 1966, 73, 16-43.

TIмм, N. M. Multivariate analysis with applications in education and psychology. Monterey: Brooks/Cole, 1975.

(Manuscript received November 31, 1981; revision accepted for publication March 30, 1982.) 